\title{
Radiological Techniques in the Diagnosis of Strangulating Small Bowel Obstruction
}

\author{
Ashwini Kumar Jha' \\ 'Department of General Surgery, Janaki Medical College Teaching Hospital, Janakpurdham, Dhanusha, Nepal.
}

\begin{abstract}
Early detection of signs of strangulation in case of acute small bowel obstruction is critical because it mandates an operation. Therefore the role of different radiological techniques used for diagnosing strangulating small bowel obstruction has been discussed here.

Strangulation remains a major problem in patients with acute small bowel obstruction. Despite of having several radiological diagnostic tools, CT is the only method with established higher diagnostic sensitivity and specificity in patients with acute small bowel obstruction as well as strangulation.
\end{abstract}

Keywords: obstruction; radiological techniques; small intestines; strangulation.

\section{INTRODUCTION}

Acute small bowel obstruction is a common surgical emergency, accounts for $12-16 \%$ of all surgical admissions in patients with acute abdominal conditions. ${ }^{1}$ Small Bowel Obstruction (SBO) is caused by variety of pathologic processes, mostly by adhesions ( $\sim 60 \%)$, followed by malignancy $(\sim 20 \%)$, hernias ( 10\%) and Crohn disease $(\sim 5 \%) .^{2}$ Its diagnosis is based on carefully taken patient history, clinical examinations and radiological findings. The major problem is the early detection of strangulation, which has a mortality of up to $40 \%$. Determining which patients require urgent operative management remains a significant challenge, due to the highly variable presentation and course of the disease. Clinical findings suspicious for strangulation such as constant abdominal pain, fever, and altered laboratory values like elevated WBC, increased serum amylase, and metabolic acidosis are often nonspecific and unreliable at differentiating simple obstruction from strangulating obstruction. Abdominal imaging plays an important role in the acute setting. It can indicate the site, level, degree and cause of obstruction and assess for the presence of ischemia. ${ }^{3}$ This article presents the various imaging techniques used in establishing the diagnosis of acute small bowel obstruction.

\section{CONVENTIONAL RADIOGRAPHY}

By 1920, plain abdominal radiography were used in the diagnosis of intestinal obstruction. ${ }^{4}$ Abdominal radiography in conjunction with the clinical examination is diagnostic in only $50-60 \%$ cases. ${ }^{1}$ Typically, conventional radiography is the first imaging procedure used in patients with bowel obstruction with a diagnostic accuracy of $46-80 \%$. Signs of small bowel obstruction on abdominal radiographs include distended bowel loops of greater than $3 \mathrm{~cm}$, collapsed colon, differential

Correspondence: Dr. Ashwini Kumar Jha, Department of General Surgery, Janaki Medical College Teaching Hospital, Janakpurdham, Dhanusha, Nepal. Email: drashwinikumariha@gmail.com, Phone: 9842494827. 
air-fluid levels, and thickened bowel wall (Figure 1a). The string of pearls sign, caused by slow resorption of intraluminal air leaving small bubbles trapped between the valvulae conniventes may also be identified (Figure $1 \mathrm{~b})$.
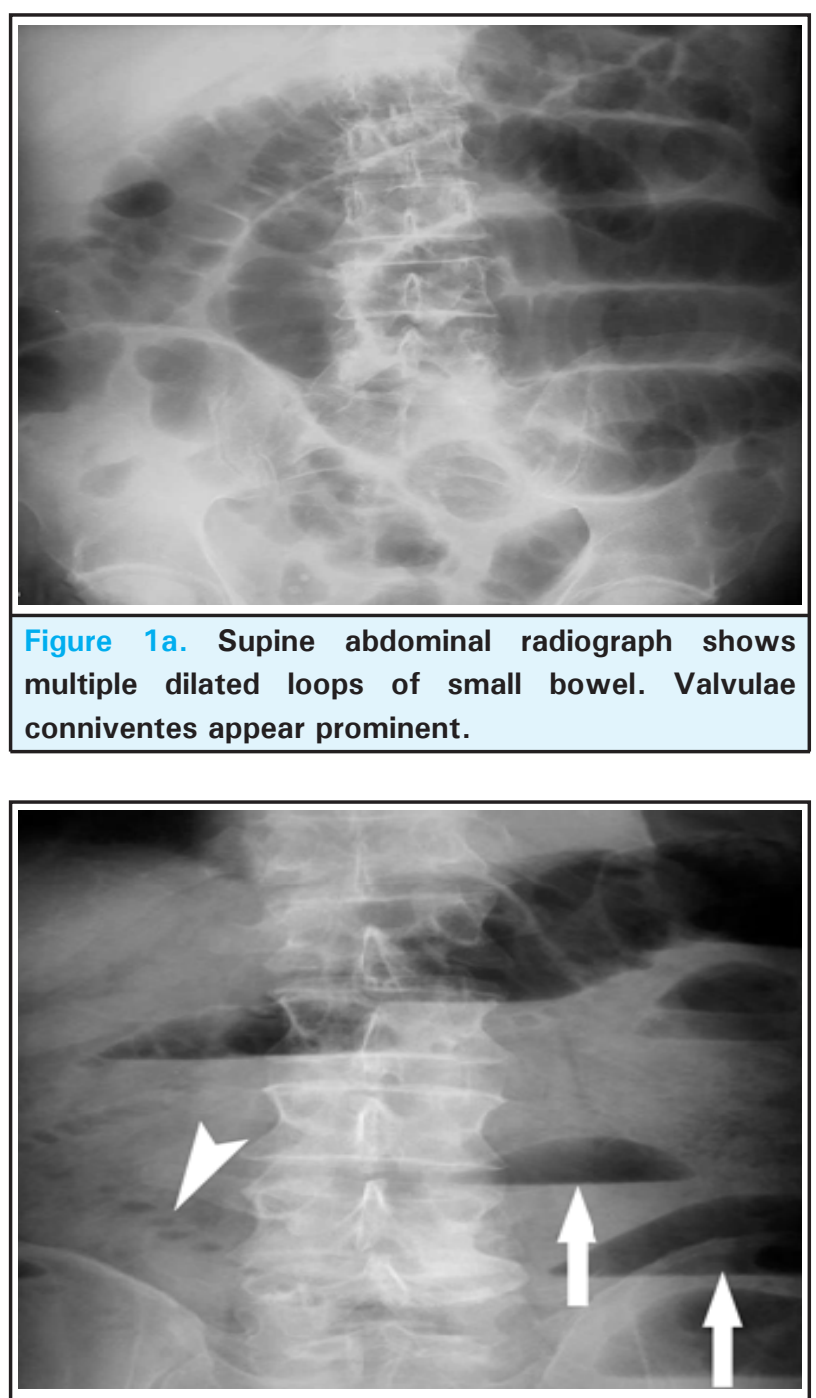

Figure 1b. Upright abdominal radiograph shows multiple air - fluid levels (arrows) and string-of-pearls sign (arrowhead).

Strangulation may be indicated by edematous folds, pneumatosis intestinalis (Figure 2a), and gas in the portal vein (Figure 2b), but these features are rarely seen.

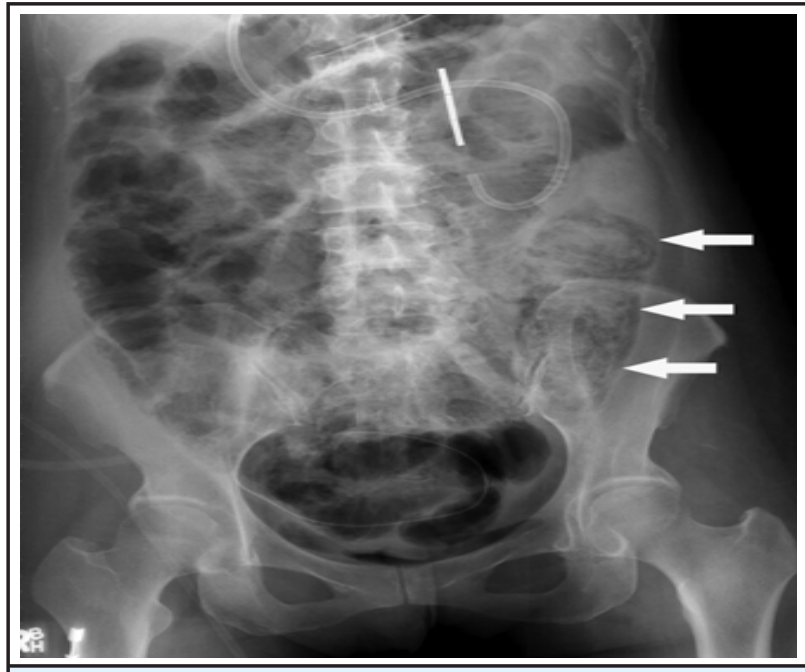

Figure 2a. Supine abdominal radiograph shows linear radiolucency (white arrows) along wall of bowel, which is consistent with pneumatosis intestinalis.

Dilated loops of small bowel are also present.

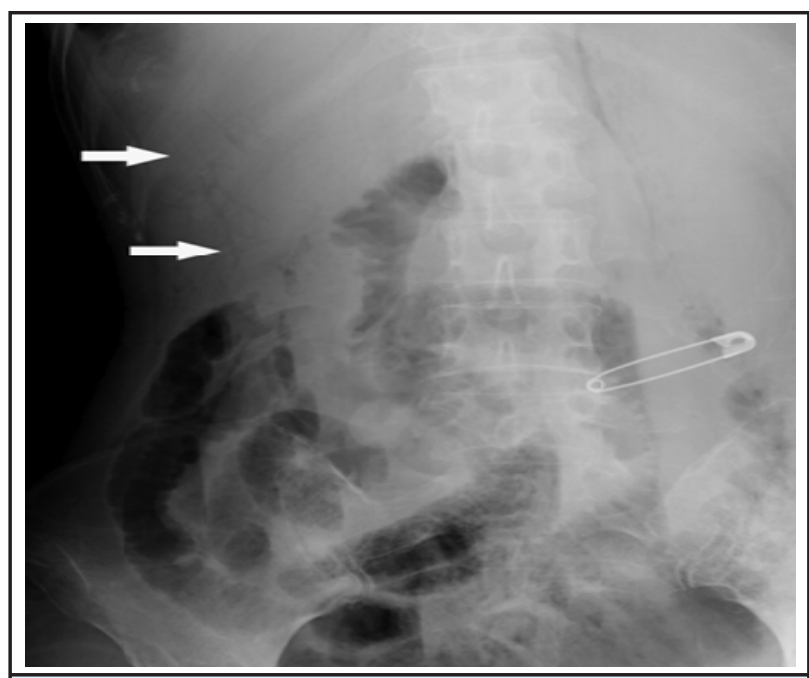

Figure 2b. Right-side-up decubitus abdominal radiograph shows multiple branching radiolucencies (white arrows) in periphery of liver shadow, which is indicative of portal venous gas. Dilated loops of small bowel are also present, which is consistent with small-bowel obstruction.

Radiographs have been shown to be sensitive for highgrade but not for low-grade obstructions, ${ }^{1}$ however, abdominal radiographs are not confirmatory or are even confusing, and the cause of obstruction is rarely detected. ${ }^{5}$ Except for inguinal hernias, ${ }^{3}$ and gallstone ileus (Figure 3), the cause of obstruction is often indiscernible on radiographs. 


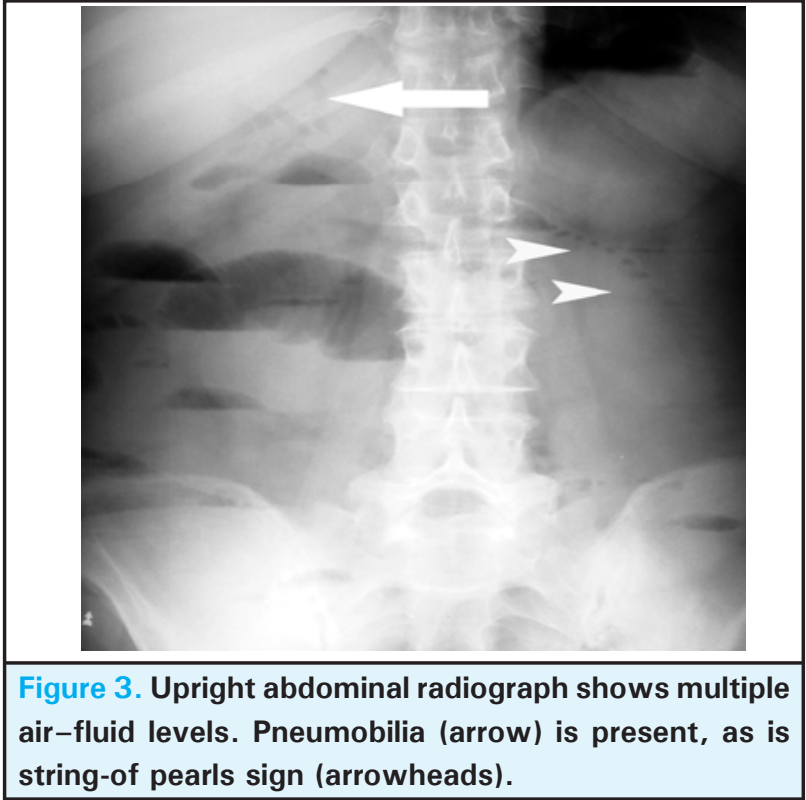

Despite its limitations, it continues to be the initial imaging examination for patients with suspected smallbowel obstruction because of its sensitivity in revealing high-grade obstruction, ${ }^{1}$ wide availability and relatively low cost.

\section{CONTRAST STUDIES}

Barium enteroclysis was introduced in 1929 in the field of radiology. Oral contrast studies such as a small-bowel follow-through may establish the diagnosis of intestinal obstruction and determine the level and cause of obstruction. Findings suggestive of obstruction include dilated loops of small bowel and a delayed transit time of barium through a transition point. ${ }^{3}$ Contrast studies, especially enteroclysis, can detect minimal adhesions, small intraluminal mass lesions and mucosal changes even when there is no bowel dilatation. ${ }^{7-11}$ It is particularly helpful in depicting and grading the severity of partial obstruction and demonstrating the sites of multifocal incomplete obstructions, and can serve as an adjunct to CT if more information, such as how much contrast material is making its way through the obstruction, is required (Figure 4). ${ }^{12}$

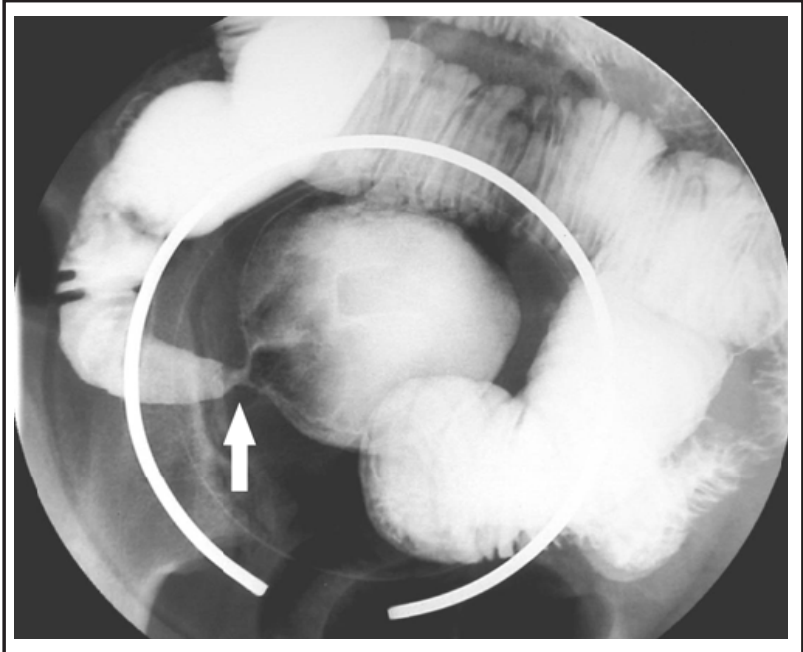

Figure 4. Enteroclysis- Spot film from enteroclysis shows small-bowel loop narrowing (white arrow) due to postoperative adhesion.

However, barium studies are unable to identify extraluminal conditions. Furthermore, the risk of concretion of barium delays further investigations required to confirm diagnosis, ultimately delaying in surgery. Because it is an invasive method and requires sedation for patients, it may not be feasible in critically ill patients in the preoperative period. Other limitations of small-bowel follow-through include the length of time required to perform the study, dilution of barium because of excess residual intraluminal fluid, and the inability of the patients to drink the barium in an acute setting. ${ }^{3}$ It should also be avoided in patients with markedly diminished intestinal peristalsis. ${ }^{6}$

\section{ULTRASONOGRAPHY}

With the advancement in technology, sonography was invented in 1950s-60s. On sonography, small bowel obstruction is suspected if multiple dilated $(>3 \mathrm{~cm})$, fluid-filled bowel loops are seen (Figure 5). 
Jha. Radiological Techniques in the Diagnosis of Strangulating Small Bowel Obstruction

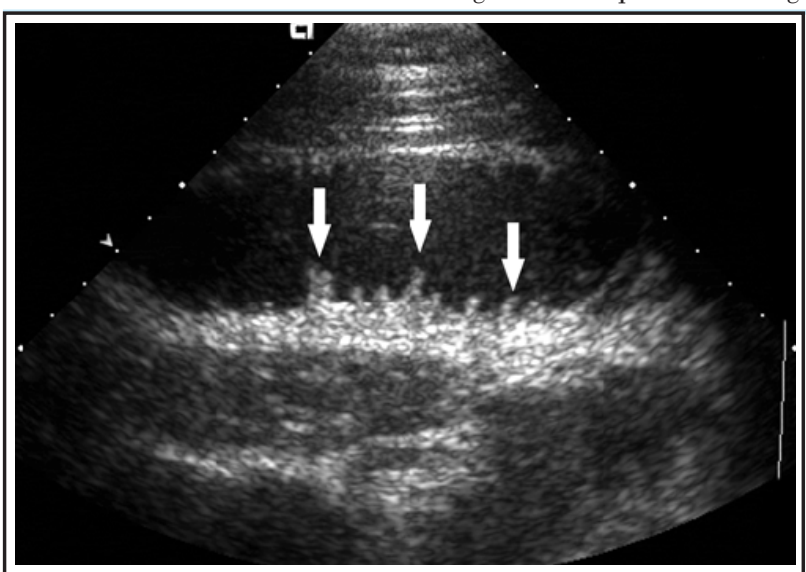

Figure 4. Abdominal sonogram shows dilated, fluidfilled loop of small bowel with prominent valvulae conniventes (white arrows).

It is able to diagnose hernia or tumor as a cause of obstruction. The presence of aperistalsis, fluid-filled bowel distension, and thickened bowel wall supports infarction in the appropriate clinical context. ${ }^{13}$ It has been reported to have a sensitivity of $89 \%$ compared with $71 \%$ for conventional radiography in diagnosing smallbowel obstruction and is superior in its ability to identify features of strangulation and to predict the location and cause of obstruction. ${ }^{13}$ It is not considered helpful in most patients with intestinal obstruction. This is easily appreciated if one remembers that the presence of abundant gas in the intestinal tract prevents satisfactory examination of the abdomen, and that adhesions, the most common cause of intestinal obstruction, are not visible on a sonogram. ${ }^{14}$ Although not routinely used, sonography may be indicated in critically ill patients as their transfer to the examination table may be time consuming and difficult. Judicious use of sonography in evaluating patients with bowel obstruction may be helpful in confirming the level of obstruction, and in identifying of the cause of obstruction, ${ }^{15}$ as well as in case of a children or in pregnant women. ${ }^{16}$

\section{COMPUTED TOMOGRAPHY (CT)}

After the invention of CT in 1979, it became the technique of choice in the diagnosis of suspected acute small bowel obstruction for several reasons. First, it does not require oral contrast material because the retained intraluminal fluid serves as a natural negative contrast agent. Second, when compared with enteroclysis, CT is rapid, noninvasive, and readily available. ${ }^{3}$ Finally, it is superior to barium studies in showing bowel wall, extraluminal masses, revealing inflammatory lesions as well as features of strangulation. ${ }^{17}$ The diagnosis of SBO on CT is made when the small bowel is dilated to a caliber of more than $2.5 \mathrm{~cm}$ with a distinct point of transition and normal caliber bowel beyond. ${ }^{18}$ The transition point often resemble a beak and is described as beak sign. Other reliable features include the stringof-pearl sign and the small-bowel feces sign. The smallbowel feces sign is a result of stasis and mixing of small bowel contents and is present in $82 \%$ of cases of small bowel obstruction. . $^{3,19}$

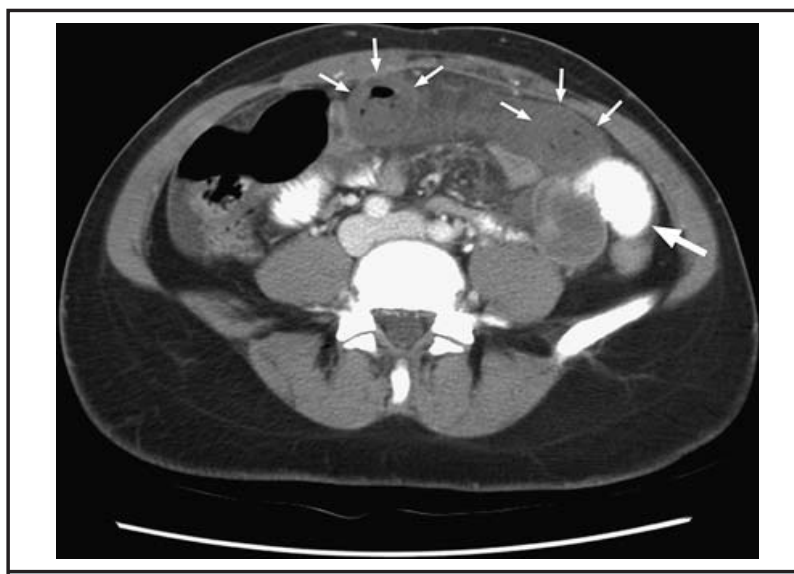

Figure 6. CT scan demonstrating reduced small bowel enhancement and wall thickening (small white arrows) in a patient with SBO and strangulation. Note that the presence of dense oral contrast obscures the assessment of bowel wall enhancement in that segment (large white arrow).

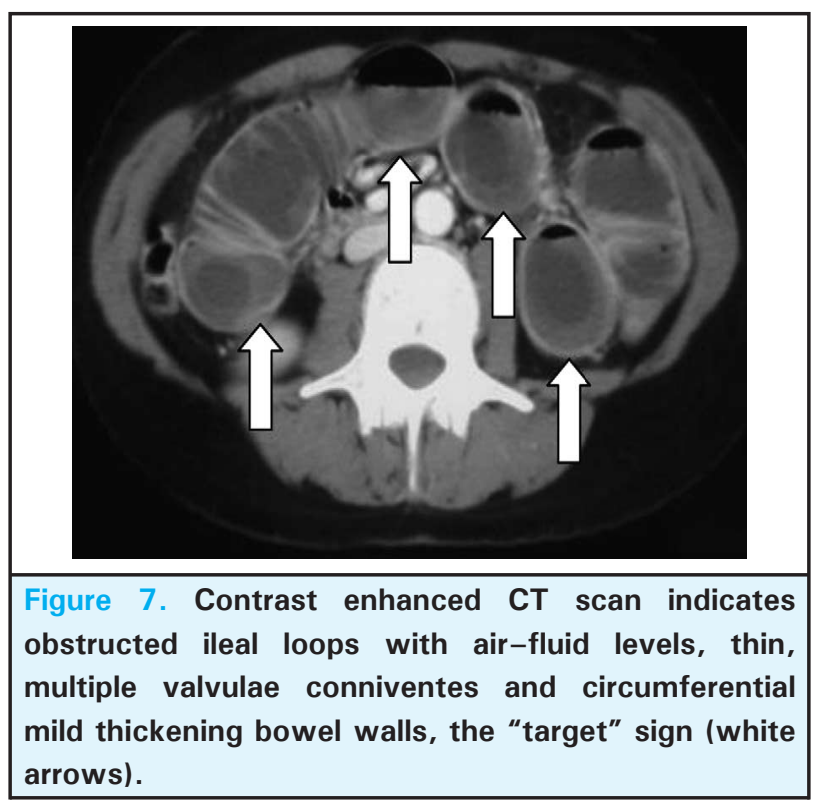

CT is not the ideal technique for diagnosis of low-grade or sub-acute obstructions and should be complemented by a contrast study, ideally enteroclysis. ${ }^{18}$ Because of its ability to demonstrate the bowel wall and mesentery, CT may differentiate simple and strangulated obstruction. Indication of strangulation on CT includes bowel and mesenteric changes of the affected loop due to an obstruction to venous flow. ${ }^{20,21} \mathrm{CT}$ signs of 
strangulations are: poor or no contrast enhancement of bowel walls (Figure 6), target sign (Figure 7), mesenteric vasculature engorgement, mesenteric congestion etc. ${ }^{22}$ One study had proved that even in the absence of classic findings like high WBC count or peritoneal signs, CT scan independently indicate bowel strangulation with a specificity of $94 \% .{ }^{23}$ Mallo et al had found CT to be $83 \%$ sensitive and $93 \%$ specific in selecting patients with ischemic bowel in the setting of SBO. ${ }^{24}$ A study conducted by Makita et al had found that mesenteric attenuation, radial distribution, and ascites, depicted on CT differentiate well between necrosis and nonnecrosis of the small bowel in patients with closed loop and strangulating obstruction. ${ }^{25}$

\section{MAGNETIC RESONANCE IMAGING (MRI)}

MRI provides rapid, accurate identification of smallbowel obstruction, ${ }^{26}$ and assists in determination of cause without exposing the patients to radiation. MRI also utilizes intraluminal air as contrast agent and is not limited by previous administration of barium. The diagnosis of small bowel obstruction on MRI involves identifying dilated loops of bowel proximal to the obstruction, a distinct transition point, and normal caliber or collapsed bowel distally. Multi-planner capabilities of MRI allow visualization of the cause of small bowel obstruction with a high degree of accuracy (Figure 8a, 8b).

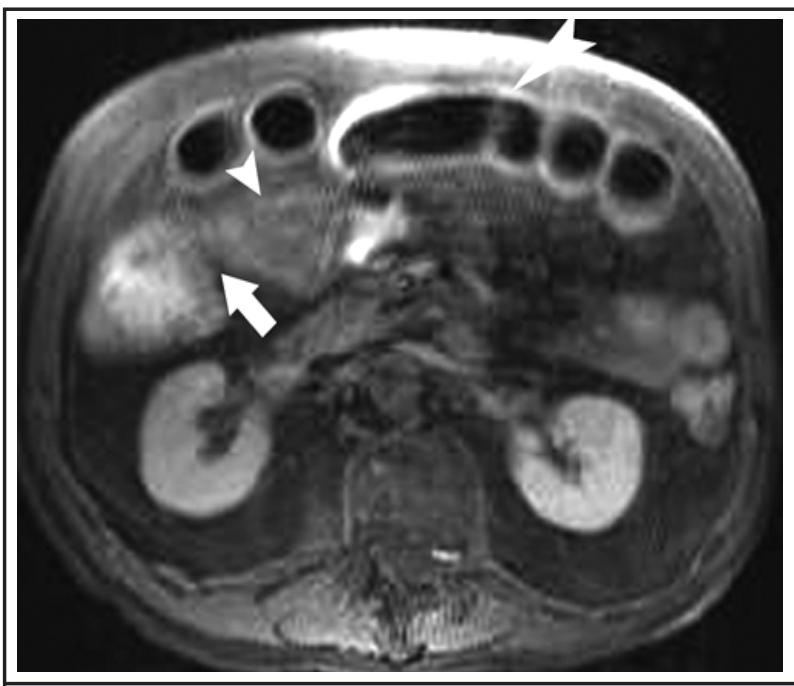

Figure 8a. MRI shows narrowing of large bowel (arrow) caused by mass (small white arrow head) with resultant proximal small bowel obstruction (large white arrow head).

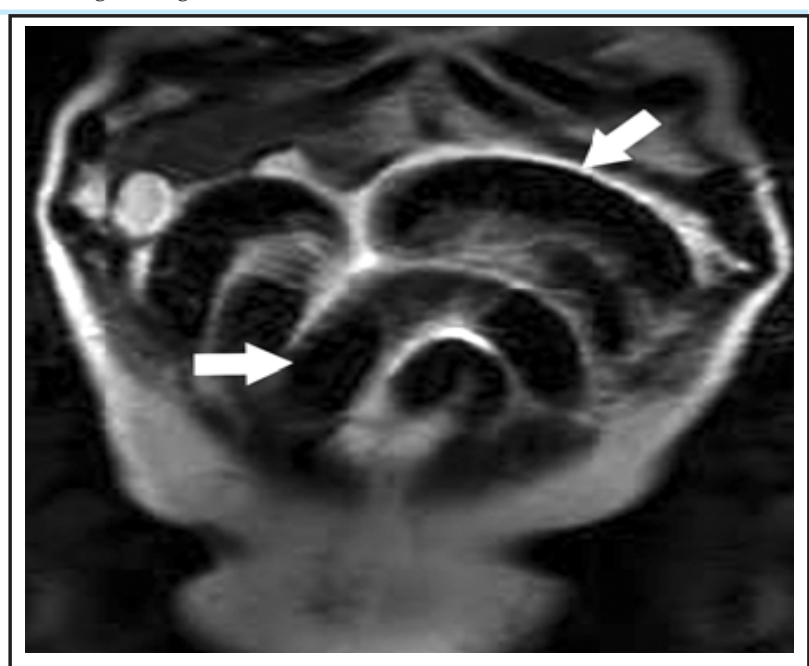

Figure 8b. Subsequent coronal image reveals numerous proximal dilated loops of small bowel (white arrows), which is consistent with diagnosis of small-bowel obstruction.

It also avoids artifacts related to peristalsis and breathing that has limited the diagnostic yield of MRI in small bowel obstruction in the past. It has a drawback of longer scanning time and inferior resolution. However, as availability and technology in MRI continues to improve, it has the potential to be an excellent diagnostic method

for evaluating small bowel obstruction.

\section{CAPSULE ENDOSCOPY}

Capsule endoscopy is currently the preferred test for mucosal imaging of the entire small intestine. ${ }^{27}$ Although, it is an easy and painless procedure permitting visualization of the entire small-bowel during its normal peristalsis, it takes a long time to pass through the entire intestine. Cheifetz et al. ${ }^{28}$ showed that capsule retention in the small intestine could help a pre-decided operation by guiding the surgeons to clearly identify the site of obstruction in patients with suspected smallbowel obstruction. Capsule retention (Figure 9) remains the major concern for physicians performing capsule endoscopy, since retention could lead to a need for an otherwise unnecessary surgery to remove the capsule. ${ }^{29}$ 


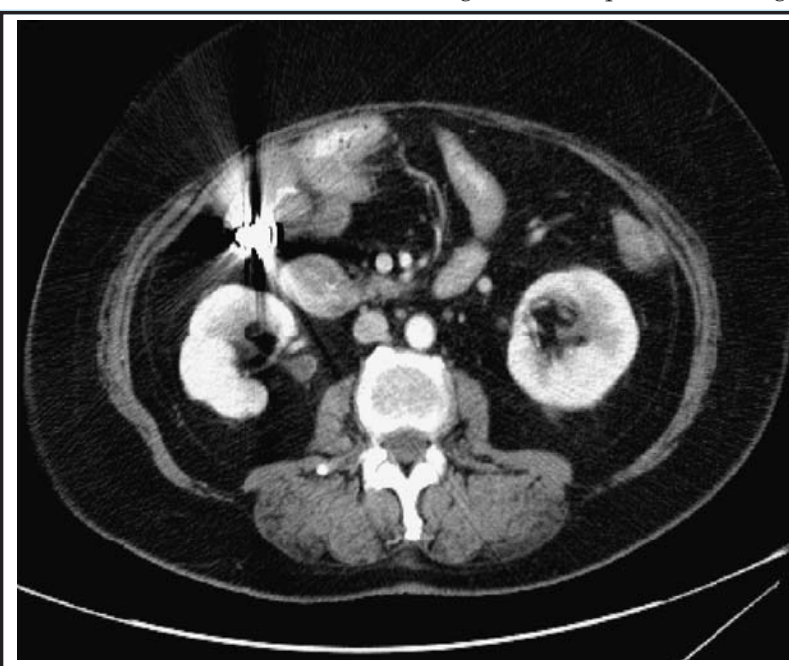

Figure 9. Axial section at distal ileum demonstrates the retained foreign body just proximal to the narrowed ileocolic anastomosis site.

Even though capsule endoscopy did not help us to diagnose a definite lesion, as we did not take clear images of the site of capsule retention, palpation of the capsule by the surgeons during operation, could lead them directly to the site of stenosis. The retained capsule can be used as a "guiding point" for a predecided surgical intervention.

\section{SUMMARY}

Despite advances in availability and accuracy of laboratory investigations and imaging techniques, clinical assessment of small bowel obstruction remains crucial. For the rapid and accurate pre-operative diagnosis of small bowel obstruction and strangulation, imaging methods can be helpful. Abdominal radiography remains the first-line imaging examination because it is readily available, cheap, and can be done serially to follow clinical progression. CT can provide additional information such as confirmation of obstruction, cause, degree and site of an obstruction and presence of ischemia. Till the further improvement of technology and introduction of other superior diagnostic methods, CT remains the choice of radiological examination for the diagnosis of strangulating SBO.

\section{REFERENCES}

1. Maglinte DDT, Heitkamp DE, Howard TJ, kelvin FM, Lappas JC. Current concepts in imaging of small bowel obstruction. Radiol Clin North Am. 2003;41(2):263-83.

2. Evers BM. Small Intestine. In: Townsend CM, (eds). Sabiston Textbook of Surgery. 17th ed. (vol. 2). Philadelphia: W.B. Saunders Company; 2004. p. 1334-42.

3. Macari M, Megibow A. Imaging of suspected acute small bowel obstruction. Semin Roentgenol. 200;36(2):108-17.

4. Welch JP. History In Bowel obstruction: Differential diagnosis and clinical management. Philadelphia, W.B. Saunders Company; 1990. p. 3.

5. Lim JH. Ultrasound of the Gastrointestinal tract, part 1, Intestinal obstruction, Springer Berlin Heidelberg. 2007. p. 27-34.

6. Herlinger H, Rubesin SE, Morris JB. Small bowel obstruction. In: Gore RM, Levine MS, eds. Textbook of gastrointestinal radiology. 2nd ed. Philadelphia, Pa: Saunders; 2000. p. 815-37.

7. Megibow AJ, Baltazar EJ, Cho KC, Medwid SW, Birnbaum BA, Noz ME. Bowel obstruction: evaluation with CT. Radiology. 1991;180 (2):313-8.

8. Fukuya T, Hawes DR, Lu CC, Chang PJ, Barloon TJ. CT diagnosis of small bowel obstruction: efficacy in 60 patients. Am J Roentgenol. 1992;158 (4):765-9.
9. Gazelle GS, Goldberg MA, Wittenberg J, Halpern EF, Pinkney L, Mueller PR. Efficacy of CT in distinguishing small bowel obstruction from other causes of small bowel dilatation. Am J Roentgenol. 1994;162 (1):43-7.

10. Maglinte DDT, Reyes BL, Harmon BH, Kelvin FM, Turner WW Jr., Hage JE, Ng AC, Chua GT, Gage SN. Reliability and role of plain film radiography and CT in the diagnosis of small bowel obstruction. Am J Roentgenol. 1996;167 (6):1451-5.

11. Makanjuola D. Computed tomography compared with small bowel enema in clinically equivocal intestinal obstruction. Clin Radiol. 1998;53(3):203-8.

12. Maglinte DDT, Balthazar EJ, Kelvin FM, Megibow AJ. The role of radiology in the diagnosis of small bowel obstruction. Am J Roentgenol. 1997;168 (5):1171-80.

13. Schmutz GR, Benko A, Fournier L, Peron JM, Morel E, Chiche L. Small bowel obstruction: role and contribution of sonography. Eur Radiol. 1997;7(7):1054-8.

14. Wilson SR. The gastrointestinal tract. In: Rumack CM, Wilson SR, Charboneau JW (eds). Diagnostic ultrasound. Mosby, St. Louis; 1991. p. 181-206.

15. Ko YT, Lim JH, Lee DH, Lee HW, Lim JW Small bowel obstruction: sonographic evaluation. Radiology. 1993;188(3):649-53. 
16. Ko YT, Lim JH, Lee DH, Yoon Y. Small bowel phytobezoars: sonographic detection. Abdom Imaging. 1993;18(3):271-3.

17. Obuz F, Terzi C, Sokmen S, Yilmaz E, Yildiz D, Fuzun M. The efficacy of helical CT in the diagnosis of small bowel obstruction. Eur J Radiol. 2003;48(3):299-304.

18. Furukawa A., Yamasaki M., Furuichi K., Yokoyama K., Nagata T, Takahashi M, Murata K, Sakamoto T. Helical CT in the diagnosis of Small bowel obstruction. Radiographics. 2001;21 (2):341-55.

19. Burkill GJC, Bell JRG, Healy JC. The utility of computed tomography in acute small bowel obstruction. Clin Radiol. 2001;56(5):350-9.

20. Ha HK, Rha SE, Kim JH, Auh YH, Maglinte DDT. CT diagnosis of strangulation in patients with small bowel obstruction: current status and future direction. Emerg Radiol. 2000;7 (1):47-55.

21. Khurana B, Ledbetter S, Mc Tavish J, Wiesner W, Ros P. Bowel obstruction revealed by multidetector CT. Am J Roentgenol. 2002;178(5):1139-44.

22. Scaglione M, Romano S, Pinto F, Flagiello F, Farina R, Acampora C, Romano L. Helical CT diagnosis of small bowel obstruction in the acute clinical setting. Eur J Radiol. 2004;50(1):15-22.
23. Jancelewicz T, Vu LT, Shawo AE, Yeh B, Gasper WJ, Harris HW. Predicting strangulated small bowel obstruction: an old problem revisited. J Gastrointest Surg. 2009;13(1):93-9.

24. Mallo RD, Salem L, Lalani T, Flum DR. Computed tomography diagnosis of ischemia and complete obstruction in small bowel obstruction: a systematic review. J Gastrointest Surg. 2005;9(5):690-4.

25. Makita O, Ikushima I, Matsumoto N, Arikawa K, Yamashita Y, Takahasi M. CT differentiation between necrotic and nonnecrotic small bowel in closed loop and strangulating obstruction. Abdom Imaging. 1999; 24:120-4.

26. Regan F, Beall DP, Bohlman ME, Khazan R,Sufi A, Schaefer DC. Fast MR imaging and the detection of small-bowel obstruction. Am J Roentgenol. 1998;170(6):1465-9.

27. Leighton JA, Goldstein J, Hirota W, Jacobson BC, Johanson $\mathrm{JF}$, Mallery JS et tal. Obscure gastrointestinal bleeding. Gastrointest Endosc. 2003;58(5):650-5.

28. Cheifetz A, Sachar D, Lewis B. Small bowel obstruction: indication or contraindication for capsule endoscopy. Gastrointest Endosc. 2004;59(5):102.

29. Kalantzis C, Apostolopoulos P, Mavrogiannis P, Theodorou D, Papacharalampous $X$, et tal. Capsule endoscopy retention as a helpful tool in the management of a young patient with suspected small-bowel disease. World J Gastroenterol. 2007;13(8):1289-91. 